EDITORIAL

GMJ.2017;6(1):1-2

www.gmj.ir

Received 2017-03-01

Accepted 2017-03-04

\title{
Communicable Diseases and Non-Communicable Diseases: Which One Is the Priority In the Health Policies?
}

\author{
Saghar Vadidi ${ }^{~}$, Mojtaba Farjam ${ }^{2 \varpi}$
}

${ }^{1}$ Student Research Committee, Fasa University of Medical Sciences, Fasa, Iran

2 Noncommunicable Diseases Research Center, Fasa University of Medical Sciences, Fasa, Iran

\section{Dear Reader,}

$\mathrm{G}$ enerally, it is believed that non-communicable diseases (NCDs) are the leading causes of mortality and morbidity in developed countries, but communicable diseases are the main challenge of health systems in non-developed parts of the world. However, in the past decades, the epidemiologic pattern of diseases has changed in developing countries $[1,2]$. In other words, many reports are showing that the main causes of mortality and morbidity in developing countries are not infectious diseases anymore.

In Iran, the good control of communicable diseases by health network during the past three decades led to significant decrease in the incidence and prevalence of these disorders [3].

The health homes in the Iranian health network in rural areas have contributed to this success [4]. Behvarzes who are local healthcare staff in rural societies has played a pivotal role in promoting the preventive measures of communicable diseases in villages $[4,5]$.

In towns and cities of Iran, health centers and health volunteers have done a lot to prevent communicable diseases. However, the success rate of this prevention was higher in rural areas. Regarding NCDs, the achievement of Iranian health system has not been as significant as expected due to the complexity of the

\section{GMJ}

2017 Galen Medical Journal

Tel/Fax: +98 7136474503

PO Box 7193616563

Email:info@gmj.ir preventive measures in NCDs of a human being. For instance, for almost all NCDs, there is no vaccination approved till now, although studies are being performed [6-8]. Moreover, when the life span increased due to the control of communicable diseases, more people reached to ages made them vulnerable to chronic NCDs as diabetes mellitus, hypertension and their health consequences [9].

Another reason for the emergence of NCDs with high prevalence in the Iranian society is the lifestyle changes especially pertinent to diet and activity [10].

However, neglecting communicable diseases is of major concern. Although infectious diseases seem to kill fewer people directly, they remain important health issues.

First of all, we should consider the emergence of disease pandemics is killing thousands of thousands of people in last twenty years, and world health organization (WHO) experienced difficulties in controlling these crises. We should not forget that there are no borders between countries protecting them against affecting people from these lethal infections.

Second, some intensive care unit (ICU) patients are admitted due to trauma or internal diseases finally die from sepsis. Viral diseases are of particular importance. New variations and continues genetic transformations in viral agents make them remain the killers of

\footnotetext{
Correspondence to:

Mojtaba Farjam MD, PhD, Noncommunicable diseases research center, , Fasa University of Medical Sciences, Ibn Sina Sq. Fasa, Iran

Telephone number: +98 715336300

Email Address: mfarjam@fums.ac.ir
} 
humans even after successful control of their previous viral forms.

Third, development of resistance of bacteria to current antibiotics is a major challenge for human health infection by so- called "bacterial superinfection" in hospitals can easily complicate simple patients with NCDs. Many chronic diseases predispose patients to these opportunistic infections. Advances in the treatment of chronic diseases and widespread use of immunosuppressive agents puts more and more patients at the risks of infectious diseases. So a great endeavor is needed to combat these antibiotic resistant bacteria, fungi, and viruses.
Furthermore, many lines of evidence show that some chronic non-communicable diseases could be attributable to infectious. Based on these evidences, cancers and autoimmune disorders are among those diseases believed to have some correlations with viral agents. The better control of infectious diseases has a major role in the prevention of NCDs.

[GMJ.2017;6(1):1-2]

Keywords: Communicable Diseases; Non-Communicable Diseases; Health Policies; world Health Organization

\section{References}

1. Askarian M, Mansour Ghanaie R, Karimi A, Habibzadeh F. Infectious diseases in Iran: a bird's eye view. Clin Microbiol Infect. 2012;18(11):1081-8.

2. Boutayeb A. The double burden of communicable and non-communicable diseases in developing countries. Trans R Soc Trop Med Hyg. 2006;100(3):191-9.

3. Moghadam MN, Sadeghi V, Parva S. Weaknesses and challenges of primary healthcare system in Iran: a review. Int J Health Plann Manage. 2012;27(2):e121-31.

4. Farjam M, Bahrami H, Bahramali E, Jamshidi J, Askari A, Zakeri H, et al. A cohort study protocol to analyze the predisposing factors to common chronic non-communicable diseases in rural areas: Fasa Cohort Study. BMC Public Health. 2016;16(1):1090.

5. Naghavi M, Shahraz S, Sepanlou SG, Dicker D, Naghavi P, Pourmalek F, et al. Health transition in Iran toward chronic diseases based on results of Global Burden of Disease 2010. Arch Iran Med. 2014;17(5):321-35.
6. Ménard J. A vaccine for hypertension. J Hypertens. 2007;25(1):41-6.

7. Farjam M, Zhang GX, Ciric B, Rostami A. Emerging immunopharmacological targets in multiple sclerosis. J Neurol Sci. 2015;358(12):22-30.

8. McKee SJ, Bergot AS, Leggatt GR. Recent progress in vaccination against human papillomavirus mediated cervical cancer. Rev Med Virol. 2015;25(S1):54-71.

9. Ostovar A, Nabipour I, Larijani B, Heshmat R, Darabi H, Vahdat K, et al. Bushehr Elderly Health (BEH) Programme, phase I (cardiovascular system). BMJ open. 2015;5(12):e009597.

10. Ghasemian A, Ataie-Jafari A, Khatibzadeh S, Mirarefin M, Jafari L, Nejatinamini S, et al. National and sub-national burden of chronic diseases attributable to lifestyle risk factors in Iran 1990 - 2013; study protocol. Arch Iran Med. 2014;17(3):146-58. 